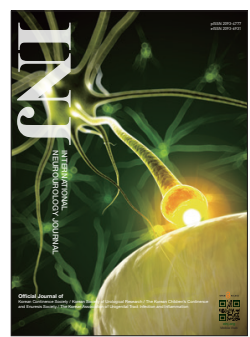

\title{
Two Fronts of Future Medicine
}

\author{
Jin Wook Kim (iD) https://orcid.org/0000-0003-4157-9365 \\ Editorial Member \\ Department of Urology, Chung-Ang University College of Medicine, Seoul, Korea \\ E-mail: jinwook.kim.md@gmail.com
}

In the age where artificial intelligence and graphene enters the common parlance of the every man, the field of neurourology seems starkly stagnant in comparison. Where is our twenty-first century technological leap forward?

\section{Catching You in the Act: Detecting the Act of Urination}

The current issue presents an original article by Whangbo et al. [1] which presents a study identifying the act of voiding by analyzing signals from 3-axis accelerometers in your smart band. While traditional nocturia experts may scoff at the lack of information provided by merely identifying the act, as it is unable to provide any data supplanting a written chart other than a time and frequency, the significance of the article is the promise of the future it brings to the ultimate and irrevocably approaching replacement of conventional methods.

While in itself, the incidence identification technology cannot replace the methods hailed by Weiss et al. [2], it shows a new technology which boasts incomparable ease of use and potential utility. The study was assessed for 30 patients over 60 days, which may seem a paltry aggregation by conventional means, yet had generated sufficient data to present a standard normal motion of urination. This particular point highlights the methods characteristic of future medical technology development studies. When big data was first introduced, researchers tripped over each other to simply expand conventional studies into the tens to hundreds of thousands enrolled. But future wearable technology presents the true power of big data application by uncovering heretofore unidentified patterns that had been lost by paucity of clinical sampling. Take, for instance, the conventional glucose tolerance test in identifying insulin resistance, and compare it to a 24 -hour blood glucose monitoring; again, take such a 24-hour glucose monitoring pattern and expand it to a week, a month, or, as in this study, 60 days [3]. The results uncover a previously unseen pattern hidden from our small peephole that is the clinic. Such is the first change that wearable technology and its data provide: a key to identifying voiding patterns that had never been observed. Expanding the patient base to well identified patient groups, a la supervised learning, voiding pattern analysis may even provide identification of particular neurourological anomalies ignored by conventional urodynamics. Coupled with other massive data repositories, such as imaging data, food intake patterns, meteorological data, new insight is guaranteed, and this is our first step.

Another important point this article highlights is the painstaking care the authors have gone to relate the basic parlance of information technology to the biomedical readers. To properly understand the method of this article, as well as future information technology, the reader should acclimatize her dictionary with terms used in time series analysis and neural networks. Future technology in this vein is sure to flood forth, and the International Neurourology Journal is all but happy to expand the boundaries of neurourological technology on all fronts. While, with the advent of convolutional neural networks have been instrumental in unleashing the sense of the fourth industrial revolution in our minds, machine learning methods have been rapidly evolving for quite some time [4]. Established methods 
of machine learning, such as support vector machines, introduced in this article as a good baseline method to compare against, should enter the language of research, at least as familiar as various statistical methods do [5]. Likewise, hurdles of machine learning, such as the vanishing gradient problem and overfitting, choices of design methods for supervised versus unsupervised learning should at least be recognized. The authors have kindly described long short-term memory algorithms and recurrent neural network structures for the reader. It should be the reader's role to accept these terms as part of the new language of research, much like learning a new language.

\section{The Culture of New Extracellular Matrix}

In 1938, the famous aviator Charles Lindbergh, along with Nobel laureate Alexis Carrel published a book "The culture of new organs" [6]. While fraught with many personal tragedies, Lindbergh wondered why his sister-in-law's heart condition could not be surgically repaired. While they were not able to grow a heart for her, they were able to develop the Carrel-Lindbergh pump, which later evolved into the heart-lung machines used in open-heart surgery.

The long and arduous development has often demoralized many researchers in the field of regenerative medicine, yet one has to understand the short span of time it took from plate culture based tissue on conventional agar to 3-dimensoinal bioprinting. In this issue, Setiawati et al. [7] reviews the recent developments in recreating extracellular matrix, highlighting the importance of scaffolds to not only mimic the physical shape, but also to mimic cellular signals.

The authors provide a broad description of tactics, both old and new. The 2 polar opposites of approach, decellularization and artificial scaffolding is shown to merge. Decellularization, with its obvious disadvantages, persist calling for a need for artificial biomaterials to step up their game. Such tactics to more fully mimic cleaned tissue is introduced here, with examples showing regulatory mimicry by expression of particular ligands and receptors. In other cases, artificial polymers are fused to enhance expression of previously unattainable levels of mimicry.

While these steps may seem small in comparison to Lindbergh's dream of attaining an artificial organ ready for trans- plant, it has been only twenty years since the human embryonic stem cell was first isolated [8]. One is also reminded that only one year after that, Atala's team had implanted a lab grown bladder in a patient [9]. The importance highlighted by the review is that we are now delving a new way forward, marrying the 2 classical schools of approach to support tissue. We may be in the future faster than we realize.

- Conflict of Interest: No potential conflict of interest relevant to this article was reported.

\section{REFERENCES}

1. Whangbo TK, Eun SJ, Jung EY, Park DK, Kim SJ, Kim CH, et al. Personalized urination activity recognition based on a recurrent neural network using smart band. Int Neurourol J 2018;22(Suppl 2):S91-100.

2. Weiss JP, Blaivas JG, Stember DS, Chaikin DC. Evaluation of the etiology of nocturia in men: the nocturia and nocturnal bladder capacity indices. Neurourol Urodyn 1999;18:559-65.

3. van Baak MA. 24-hour glucose profiles on diets varying in protein content and glycemic index. Nutrients 2014;6:3050-61.

4. Gulshan V, Peng L, Coram M, Stumpe MC, Wu D, Narayanaswamy A, et al. Development and validation of a deep learning algorithm for detection of diabetic retinopathy in retinal fundus photographs. JAMA 2016;316:2402-10.

5. Mannini A, Trojaniello D, Cereatti A, Sabatini AM. A machine learning framework for gait classification using inertial sensors: application to elderly, post-stroke and Huntington's disease patients. Sensors (Basel) 2016;16(1). pii: E134. https://doi.org/10.3390/s16010134.

6. Carrel A, Lindbergh CA. The culture of whole organs. Science 1935;81:621-3.

7. Setiawati A, Nguyen HT, Jung Y, Shin K. Future research directions in the design of versatile extracellular matrix in tissue engineering. Int Neurourol J 2018:22(Suppl 2):S66-75.

8. Itskovitz-Eldor J. 20th Anniversary of isolation of human embryonic stem cells: a personal perspective. Stem Cell Reports 2018;10:1439-41.

9. Rothberg MB, Atala A. History and development of regenerative medicine and tissue engineering in urology. In: Patel S., Moran M., Nakada S. editors. The history of technologic advancements in urology. Cham(Switzerland): Springer: 2018. p. 289-317. 